\title{
Reduced pulmonary blood flow in regions of injury 2 hours after acid aspiration in rats
}

\author{
Torsten Richter ${ }^{1 *}$, Ralf Bergmann², Guido Musch³ ${ }^{3}$ Jens Pietzsch ${ }^{2,4}$ and Thea Koch ${ }^{1}$
}

\begin{abstract}
Background: Aspiration-induced lung injury can decrease gas exchange and increase mortality. Acute lung injury following acid aspiration is characterized by elevated pulmonary blood flow (PBF) in damaged lung areas in the early inflammation stage. Knowledge of PBF patterns after acid aspiration is important for targeting intravenous treatments. We examined PBF in an experimental model at a later stage (2 hours after injury).

Methods: Anesthetized Wistar-Unilever rats $(n=5)$ underwent unilateral endobronchial instillation of hydrochloric acid. The PBF distribution was compared between injured and uninjured sides and with that of untreated control animals $(n=6)$. Changes in lung density after injury were measured using computed tomography $(C T)$. Regional PBF distribution was determined quantitatively in vivo 2 hours after acid instillation by measuring the concentration of $\left[{ }^{68} \mathrm{Ga}\right]$-radiolabeled microspheres using positron emission tomography.

Results: CT scans revealed increased lung density in areas of acid aspiration. Lung injury was accompanied by impaired gas exchange. Acid aspiration decreased the arterial pressure of oxygen from $157 \mathrm{mmHg}$ [139;165] to $74 \mathrm{mmHg}[67 ; 86]$ at 20 minutes and tended toward restoration to $109 \mathrm{mmHg}[69 ; 114]$ at 110 minutes $(P<0.001)$. The PBF ratio of the middle region of the injured versus uninjured lungs of the aspiration group (0.86 [0.7;0.9], median $[25 \% ; 75 \%]$ ) was significantly lower than the PBF ratio in the left versus right lung of the control group (1.02 $[1.0 ; 1.05] ; P=0.016)$.

Conclusions: The PBF pattern 2 hours after aspiration-induced lung injury showed a redistribution of PBF away from injured regions that was likely responsible for the partial recovery from hypoxemia over time. Treatments given intravenously 2 hours after acid-induced lung injury may not preferentially reach the injured lung regions, contrary to what occurs during the first hour of inflammation.

Please see related article: http://dx.doi.org/10.1186/s12871-015-0014-z.

Keywords: Acute lung injury, Respiratory aspiration, Positron emission tomography, Pulmonary circulation, Pulmonary perfusion, Adult respiratory distress syndrome
\end{abstract}

\section{Background}

Lung aspiration is a common complication at the induction of general anesthesia and in emergency medicine. Lung atelectasis, alveolar edema, peribronchial hemorrhage, and neutrophil migration are consequences of an aspiration event $[1,2]$ and may lead to impaired gas exchange and extended hospital stays. In addition, acid aspiration is one of the common causes of the development of acute respiratory distress syndrome, with a mortality rate of approximately $40 \%$ [3-5].

\footnotetext{
* Correspondence: torsten.richter@uniklinikum-dresden.de

${ }^{1}$ Department of Anesthesia and Intensive Care, Carl Gustav Carus University

Hospital, Technische Universität Dresden, Dresden, Germany

Full list of author information is available at the end of the article
}

To date, lung inflammation has been treated with antibiotics and anti-inflammatory drugs such as steroids [6], with the aim of attenuating the inflammatory response as early as possible. Based on the present data, steroid therapy is not recommended after acid aspiration [2]. Targeted drug delivery would be a step toward treating lung inflammatory processes more specifically. Experimental interventions to treat aspiration have included the use of a hyperosmolar solution [7], a hypertonic saline solution [8], pentoxifylline [9], concentrated human albumin and furosemide [10]. These treatments were administered intravenously and, therefore, their delivery to injured areas was dependent on regional pulmonary perfusion. Consequently, knowledge of 
the blood flow pattern in the lungs after an aspiration event is critical for targeting the delivery of treatments to areas where they are most needed.

The distribution of pulmonary blood flow (PBF) increased in the injured lung regions of rats 10 minutes after the administration of hydrochloric acid $(\mathrm{HCl})$ [11], and this PBF distribution pattern remained within the first hour after acid aspiration [12], which was within the time frame of the first peak of the inflammatory response in the rats [13]. Furthermore, the lung weight constantly increased within the first two hours after aspiration [14], indicating edema formation due to changes in lung microvascular permeability [10].

The manner by which the PBF distribution changes as an ensuing inflammatory process evolves has not been established. Therefore, we non-invasively characterized the spatial PBF distribution in rats 2 hours after acidinduced lung injury using $\left[{ }^{68} \mathrm{Ga}\right]$-radiolabeled microspheres and small animal positron emission tomography (PET). The focus of this study was on evaluating the PBF distribution pattern in areas of lung injury in a later stage of acid-induced acute lung injury. PBF in these areas was specifically compared with PBF in uninjured areas and in control animals. The clinical relevance of this study lies in its answer to the question of whether, in this stage of aspiration-induced lung injury, damaged areas will still be preferentially perfused.

\section{Methods}

\section{Experimental setting}

All of the experiments were conducted using 11 male Wistar-Unilever rats (mean body weight, 355 g; range 339-381 g) according to the German Regulations for Animal Welfare. The protocol was approved by the local Ethics Committee for Animal Experiments (Landesdirektion Dresden, 24D-9168.11-4/2007-1).

In each experiment, rats were anesthetized using a combination of desflurane (7-12\%) and a single intraperitoneal injection of ketamine $(75 \mathrm{mg} / \mathrm{kg})$. Local anesthesia during the operation was provided by infiltration with $1 \%$ lidocaine. Body temperature and supine position were maintained by placing the animals in a custom-built, heated, plastic tube. Catheters were inserted into the femoral artery and femoral vein for the following purposes: to measure arterial blood pressure and heart rate (Model 54S, Component Monitoring System; Hewlett-Packard, Saronno, Italy), to obtain arterial blood samples for blood gas analyses (ABL 50, Radiometer, Copenhagen, Denmark), and to provide intravenous infusions. To administer $\mathrm{HCl}$ to the lung, we inserted a cannula after tracheostomy.

\section{Experimental protocol}

The anesthetized, spontaneously breathing animals were allowed to stabilize after preparation and were then randomly assigned to the control group $(n=6)$ or the injury group $(n=5)$, and baseline values were recorded. In both groups, a catheter was blindly inserted to the maximum depth through the tracheostomy tube into either the left or right lung. Only in the injury group was acid aspiration induced by unilateral intra-bronchial administration of $0.1 \mathrm{M} \mathrm{HCl}(0.4 \mathrm{~mL} / \mathrm{kg}$ body weight $)$ through the catheter. The amount of $\mathrm{HCl}$ administered into the supinepositioned animals was limited to prevent life-threatening lung failure and spillover to the contralateral lung. The control group received nothing through the catheter.

Physiological parameters (including blood gases and hemodynamics) were determined at baseline $\left(\mathrm{T}_{0}\right)$ and then $20\left(\mathrm{~T}_{20}\right)$ and $110\left(\mathrm{~T}_{110}\right)$ minutes after acid aspiration or baseline measurements of the injury and control group, respectively. Computed tomography (CT) was used to identify regions of increased pulmonary density after aspiration. Spatial PBF was quantitatively determined using radiolabeled microspheres and PET. Figure 1 shows the protocol schema.

\section{Small animal computed tomography imaging}

The degree of injury was assessed using lung density measurements obtained from 10-minute CT scans of the lung, which were acquired at baseline $\left(\mathrm{T}_{0}\right)$ and at $\mathrm{T}_{110}$ using a micro-CT scanner (Skyscan 1178 micro-CT system, Kontich, Belgium) with a resolution of $0.083 \mathrm{~mm}$ and the following specifications: $83-\mu \mathrm{m}$ pixel size, 360 -degree scanning, average of 2 frames, $85-\mathrm{mm}$ field of view, 9 minute 7 second scanning time, $65-\mathrm{kV}$ voltage, and $615-\mu \mathrm{A}$ current. The data were exported and converted to the ECAT2 format.

\section{Radiolabeling of microspheres}

Human serum albumin microspheres with a mean diameter of $20 \mu \mathrm{m}$ were labeled with gallium- $68\left({ }^{68} \mathrm{Ga}\right)$, as previously described [15], to obtain injectable ${ }^{68} \mathrm{Ga}$-human serum albumin microspheres (Ga-68 microspheres).

\section{Small animal positron emission tomography imaging}

A microPET ${ }^{\circ}$ P4 scanner (Siemens Preclinical Solutions, Knoxville, TN, USA) with a spatial resolution of $1.85 \mathrm{~mm}$ full-width half maximum in the center of the field of view along both the tangential and radial directions was used. Following a 10-minute transmission scan, a 1-minute bolus infusion of Ga-68 microspheres was initiated, and a 30minute emission scan was performed. Emission scans were corrected for decay and attenuation, and the frames were reconstructed using ordered subset expectation maximization. The voxel size was $0.8 \times 0.8 \times 1.2 \mathrm{~mm}^{3}$.

\section{Image processing \\ Registration}

Image files were processed using the ROVER software (ABX $\mathrm{GmbH}$, Radeberg, Germany). CT scans were imported in 


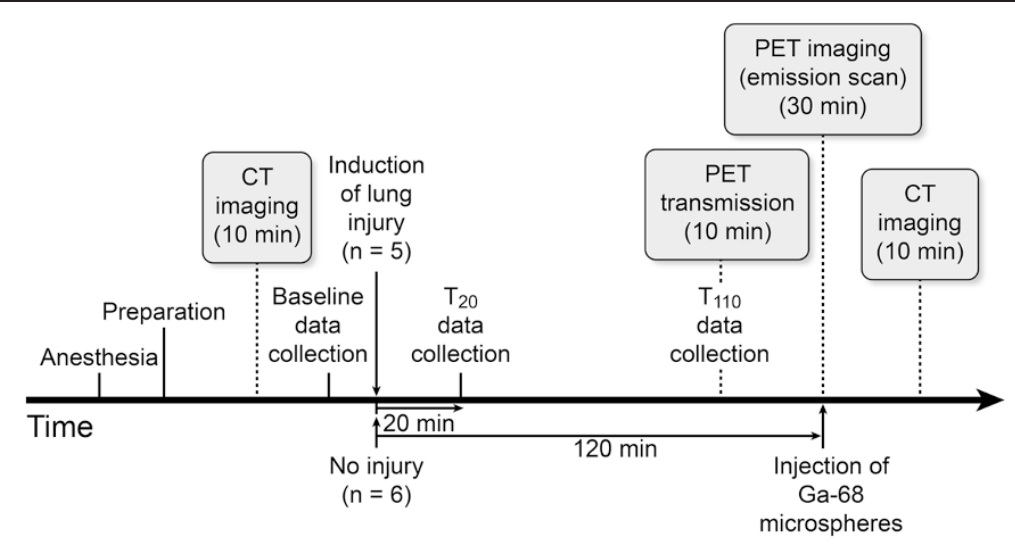

Figure 1 Study protocol schema.

Dicom format and converted to the same voxel size as the PET scans. Residual differences between images were corrected using the ROVER registration module to obtain the same alignment for all 3 dimensions of the PET and CT images. The first and second CT scans of each animal were analyzed for changes in lung density. Poorly aerated and nonaerated areas, which were visually identified, were assumed to be areas of lung injury [16].

\section{ROI definition}

Masks that described the volume for the regions of interest (ROIs) were created and placed over the coregistered PET scan of each animal. The ROI corresponding to the entire lung field on the PET image $\left(\mathrm{ROI}_{\text {total }}\right)$ was divided into 2 sections along the sagittal plane, resulting in 2 separate ROIs ( $\mathrm{ROI}_{\text {lung side }}$ ) for the right $\left(\mathrm{ROI}_{\text {right }}\right)$ and left $\left(\mathrm{ROI}_{\text {left }}\right)$ lungs (Figure 2). Masks were created to define transverse layers as representative subregions of the apical, middle, and caudal regions of the lung (Figure 2). The resulting ROIs ( $\mathrm{ROI}_{\text {apical, }}$ $\left.\mathrm{ROI}_{\text {middle, }} \mathrm{ROI}_{\text {caudal }}\right)$ were identically placed over the coregistered PET scan of each animal to compare the PET measurements between the apical, middle, and caudal regions of each lung. A spherical ROI mask was symmetrically placed on each lung side (between the middle and dorsal third of the sagittal axis, the centric and the lateral third at the transversal axis and the middle and caudal region along the vertical axis). Empirically, this standard position included the most frequently damaged lung regions after acid-induced lung injury, as observed previously in this aspiration model [11]. Areas of increased lung density following aspiration were identified on the CT scan and could be marked with this spherical ROI. The spherical ROI mask was identically placed over all co-registered CT and PET scans to define the $\mathrm{ROI}_{\text {specific }}$ $\left(0.24 \mathrm{~cm}^{3}\right)$, which was used to assess the ratios of the mean lung density in the injured versus non-injured lungs from the CT scan and to determine the PBF from the PET scan (Figure 2).

\section{Pulmonary blood flow}

The PBF distribution was derived by analyzing the pulmonary distribution of Ga-68 microspheres within the ROIs [15]. When using this method, regional perfusion is proportional to the number of microspheres that reach the ROI. Regional PBF is quantified as the fractional flow to the ROI relative to the $\mathrm{ROI}_{\text {total }}$. Regional PBF was analyzed from the selected ROI ( $\mathrm{ROI}_{\text {lung side, }}$ $\mathrm{ROI}_{\text {specific }}, \mathrm{ROI}_{\text {apical }}, \mathrm{ROI}_{\text {middle, }} \mathrm{ROI}_{\text {caudal }}$ ) (Figure 2) for each lung in the control and injury groups. The maximum standardized uptake value $\left(\mathrm{SUV}_{\max }\right)$ was calculated [11] with respect to the ${ }^{68} \mathrm{Ga}$ half-life within the ROIs to quantitatively compare PBF patterns. The PBF in each region was analyzed as the ratio of $\mathrm{PBF}$ on the injured side to that on the uninjured side in the aspiration group and as the ratio of PBF on the left side to that on the right side in the control animals.

\section{Statistical analysis}

All of the data are expressed as median values and $25^{\text {th }}$ and $75^{\text {th }}$ percentiles (median [25-75\%]) unless otherwise indicated.

Changes over time were analyzed globally using the Friedman test for each time series, followed by a paired $t$-test for individual comparisons with baseline. An unpaired $t$-test with Welch's correction was used to compare the 2 groups with respect to hemodynamic values and SUVs at individual time points. A nonparametric Wilcoxon 2-tailed, matched-pairs signed rank test was used for data comparison within groups. Calculations were performed using GraphPad Prism 5.02 for Windows (GraphPad Software Inc., San Diego, CA, USA). Statistical significance was set at $P<0.05$. 


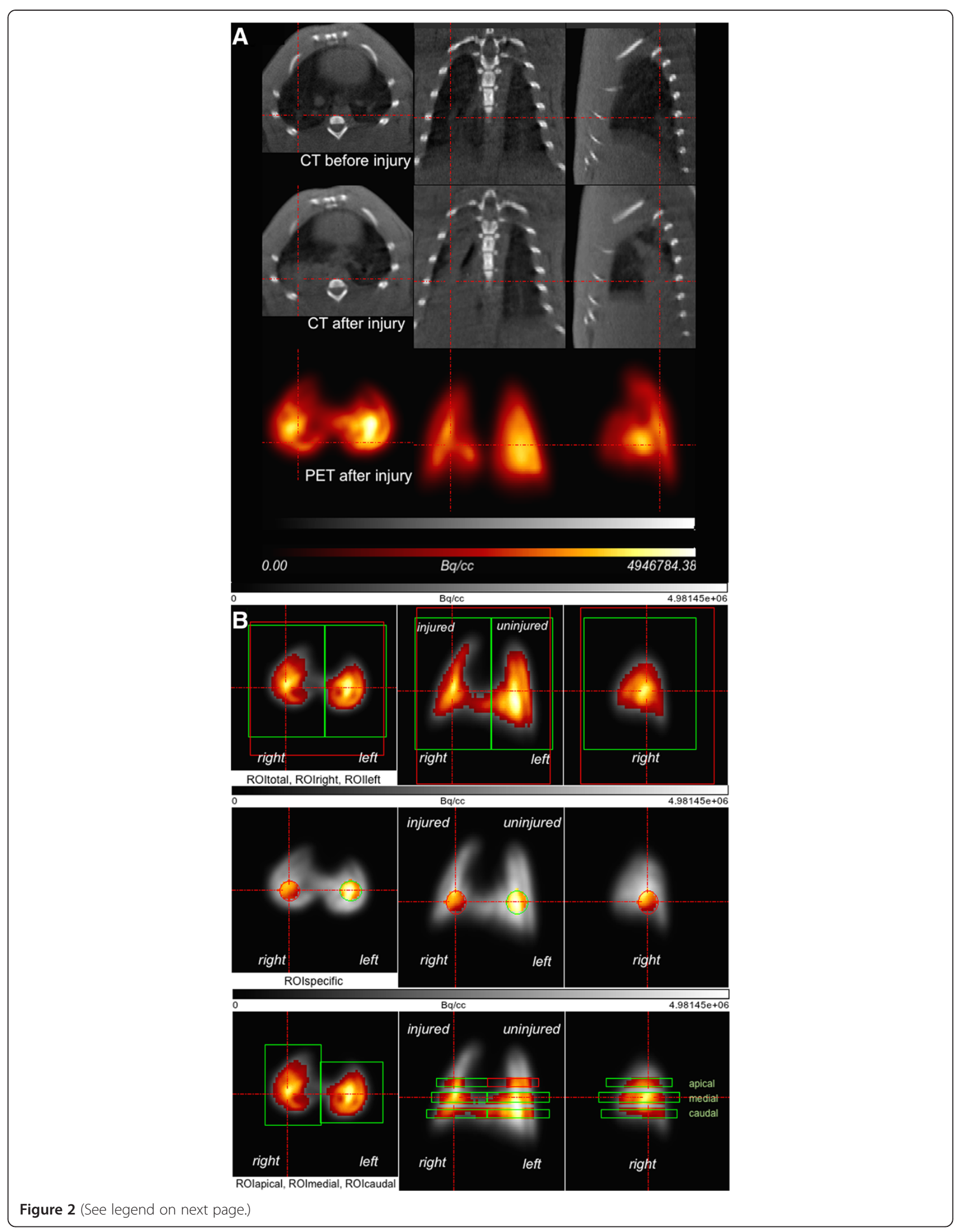


(See figure on previous page.)

Figure 2 Example of image processing after co-registration. (A) Co-registered PET and CT images in the transverse, coronal, and sagittal planes of one animal; the CT image before and after injury and the corresponding PET scan image are shown. Dark areas in the CT scan represent aerated zones. The areas of high density were formed in the right lung after injury (red lines). The PET scan shows the distribution of PBF in the left and right lungs. (B) PET images and definition of the ROI for one lung in the transverse, coronal, and sagittal planes. The tracer activity in the lung is indicated in grayscale, from lowest (black) to highest activity (white). ROI masks were used for the threshold analysis and to calculate the median maximum standardized uptake values $\left(S U V_{\max }\right)$ for each side of the lung in a 3-dimensional environment. The thresholds in the ROI masks for the entire lung (red: ROI total), the left and right lung (green), the spherical masks $\left(\mathrm{ROI}_{\text {specific }}\right)\left(0.24 \mathrm{~cm}^{3}\right)$ and the $6 \mathrm{ROIs}(\mathrm{apical}$, medial, and caudal) are indicated by the highlighted regions of the color-coded areas. Color coding shows a relative scale in which the measurements are normalized to their mean values and represent regional tracer concentrations of Ga-68 microspheres from high (white) to low (black), as determined by PET in $\mathrm{Bq} / \mathrm{cm}^{3}$. Red lines are focused on the area of injury.

\section{Results}

\section{Physiological variables}

Global physiological data are shown in Table 1. The physiological variables did not differ between the control and aspiration groups at baseline and did not change significantly during the study period in the control animals.

Table 1 Physiological variables

\begin{tabular}{|c|c|c|c|}
\hline & $T_{0}$ & $T_{20}$ & $\mathrm{~T}_{110}$ \\
\hline \multicolumn{4}{|c|}{$\mathrm{PaO}_{2}, \mathrm{mmHg}$} \\
\hline Control & $152[152 ; 160]$ & $148[141 ; 170]$ & $124[111 ; 163]$ \\
\hline Injury & 157 [139;165] & $74[67 ; 86]^{a, b}$ & $109[69 ; 114]^{a}$ \\
\hline \multicolumn{4}{|c|}{$\mathrm{PaCO}_{2}, \mathrm{mmHg}$} \\
\hline Control & $53[51 ; 61]$ & $54[53 ; 59]$ & $54[53 ; 64]$ \\
\hline Injury & $59[56 ; 62]$ & $57[50 ; 63]$ & $47[43 ; 68]$ \\
\hline \multicolumn{4}{|l|}{$\mathrm{pH}$} \\
\hline Control & $7.36[7.33 ; 7.37]$ & $7.35[7.33 ; 7.35]$ & $7.35[7.29 ; 7.36]$ \\
\hline Injury & $7.31[7.26 ; 7.36]$ & $7.46[7.3 ; 7.46]$ & $7.31[7.27 ; 7.39]$ \\
\hline \multicolumn{4}{|c|}{$\mathrm{SpaO}_{2}, \%$} \\
\hline Control & 99.3 [99.3;99.4] & 99.3 [99.2;99.4] & $98.8[98.3 ; 99.3]$ \\
\hline Injury & $99.3[99.1 ; 99.5]$ & $94.5[92.8 ; 96.4]^{\mathrm{a}, \mathrm{b}}$ & $98.2[93.1 ; 98.4]$ \\
\hline \multicolumn{4}{|c|}{ HR, beats/min } \\
\hline Control & 294 [276;324] & 319 [292;373] & 306 [295;339] \\
\hline Injury & 293 [256;326] & 303 [278;326] & 299 [273;331] \\
\hline \multicolumn{4}{|c|}{ MAP, $\mathrm{mmHg}$} \\
\hline Control & $93[91 ; 102]$ & $100[90 ; 102]$ & 95 [86;98] \\
\hline Injury & $99[85 ; 102]$ & 90 [83;91] & $83[69 ; 87]^{b}$ \\
\hline \multicolumn{4}{|c|}{ RR, breaths/min } \\
\hline Control & $59[58 ; 63]$ & $59[56 ; 62]$ & $61[55 ; 64]$ \\
\hline Injury & $57[53 ; 66]$ & $60[58 ; 71]$ & $63[61 ; 66]$ \\
\hline \multicolumn{4}{|l|}{$\mathrm{T},{ }^{\circ} \mathrm{C}$} \\
\hline Control & $35.6[35.2 ; 35.9]$ & $35.7[34.8 ; 36]$ & $36[35 ; 36.8]$ \\
\hline Injury & $35.1[34.4 ; 35.4]$ & $35.1[34.7 ; 36]$ & $36[35.2 ; 36.6]$ \\
\hline
\end{tabular}

Physiological variables at certain time points in the control group $(n=6)$ and injury group $(n=5)$. Values represent the median [interquartile range]. HR, heart rate; MAP, mean arterial blood pressure; $\mathrm{PaO}_{2}$, arterial oxygen tension; $\mathrm{PaCO}_{2}$, arterial carbon dioxide tension; $\mathrm{pH}$, arterial $\mathrm{pH}$; RR, respiratory rate; $\mathrm{SpaO}_{2}$, arterial oxygen saturation; $\mathrm{T}$, body temperature; $\mathrm{T}_{0}$, baseline; $\mathrm{T}_{20}$, 20 minutes after acid aspiration (injury group) or after baseline (control group); $\mathrm{T}_{110}, 110$ minutes after acid aspiration (injury group) or after baseline (control group). ${ }^{\mathrm{a}} P<0.05$ vs. $\mathrm{T}_{0} ;{ }^{\mathrm{b}} P<0.05$, Control vs. Injury.
Acid aspiration worsened pulmonary gas exchange, decreasing arterial partial pressure of oxygen $\left(\mathrm{PaO}_{2}\right)$ and arterial oxygen saturation $\left(\mathrm{SpaO}_{2}\right)$ values; the greatest impairment occurred after 20 minutes, and the values tended toward recovery at 110 minutes. In the aspiration group, the mean arterial pressure decreased from $99 \mathrm{mmHg}$ $[85 ; 102]$ to $90 \mathrm{mmHg}[83 ; 91]$ at 20 minutes $\left(\mathrm{T}_{20}\right)(P=0.06)$ and to $83 \mathrm{mmHg}[69 ; 87]$ at 110 minutes $\left(\mathrm{T}_{110}\right)(P=0.04)$.

\section{Density changes}

An increase in CT-derived lung density (comparing the CT scan at baseline with the CT scan after injury) allowed localization of the effects of acid administration on the lung tissue. In the injury group, $\mathrm{ROI}_{\text {specific }}$ analysis revealed localized increased density in the left $(n=4)$ or right $(n=1)$ lung. The ratio of the mean lung density in the injured versus non-injured lungs (1.4 [1.2-1.5]) was significantly higher than the ratio of lung density in the left versus right lungs in the control group (1.1 [1.0-1.1], $P=0.006)$.

\section{Regional pulmonary blood flow}

Table 2 shows the PBF data for the following regions: the entire lung field $\left(\mathrm{ROI}_{\text {total }}\right)$, right lung, left lung, $\mathrm{ROI}_{\text {specific, }}$ and 3 transverse planes (apical, middle, and caudal) of the injured and uninjured lungs. The PBF ratios in the injured versus uninjured lungs of the aspiration group were significantly lower than those in the left versus right lungs of the control group when PBF was referenced to the entire lung ( $\mathrm{ROI}_{\text {lung side }}$ ), $\mathrm{ROI}_{\text {specific }}$ (Figure 3), and $\mathrm{ROI}_{\text {middle }}$ (Figure 4). The PBF ratios of $\mathrm{ROI}_{\text {specific }}$ from the current study and a previous study [11] are shown in Figure 5.

\section{Discussion}

Using small animal imaging techniques, we quantitatively demonstrated the effects of acid aspiration on PBF in anesthetized, spontaneously breathing rats 2 hours after injury. Lung injury increased the density of the damaged regions and was accompanied by impairments in physiological variables. The regional response 2 hours after acid aspiration consisted of decreased PBF in injured lung regions accompanied by attenuation of hypoxemia and residual hypotension. 
Table 2 Pulmonary blood flow values for all regions of interest

\begin{tabular}{|c|c|c|c|}
\hline & Control group & & Injury group \\
\hline Entire lung & $111[107 ; 115]$ & & $121[108 ; 137]$ \\
\hline \multicolumn{4}{|l|}{$\mathrm{ROI}_{\text {lung side }}$} \\
\hline \multirow[t]{2}{*}{ Left side } & $110[107 ; 115]$ & Injured side & $101[94 ; 129]$ \\
\hline & $P=0.16$ & & $P=0.125$ \\
\hline Right side & $106[103 ; 111]$ & Uninjured side & $117[108 ; 135]$ \\
\hline \multicolumn{4}{|l|}{$\mathrm{ROI}_{\text {specific }}$} \\
\hline \multirow[t]{2}{*}{ Left side } & $110[107 ; 113]$ & Injured side & 98 [92;123] \\
\hline & $P=0.06$ & & $P=0.06$ \\
\hline Right side & $106[104 ; 109]$ & Uninjured side & 117 [107;135] \\
\hline \multicolumn{4}{|l|}{$\mathrm{ROI}_{\text {apical }}$} \\
\hline \multirow[t]{2}{*}{ Left side } & 92 [90;100] & Injured side & $95[71 ; 113]$ \\
\hline & $P=0.56$ & & $P=0.31$ \\
\hline Right side & $98[93 ; 101]$ & Uninjured side & 97 [92;115] \\
\hline \multicolumn{4}{|l|}{$\mathrm{ROI}_{\text {middle }}$} \\
\hline \multirow[t]{2}{*}{ Left side } & $107[104 ; 111]$ & Injured side & $96[83 ; 119]$ \\
\hline & $P=0.22$ & & $P=0.06$ \\
\hline Right side & 104 [103;108] & Uninjured side & 114 [108;133] \\
\hline \multicolumn{4}{|l|}{$\mathrm{ROI}_{\text {caudal }}$} \\
\hline \multirow[t]{2}{*}{ Left side } & 110 [105;115] & Injured side & $101[94 ; 116]$ \\
\hline & $P=0.06$ & & $P=0.19$ \\
\hline Right side & $104[100 ; 111]$ & Uninjured side & 112 [103;132] \\
\hline
\end{tabular}

Pulmonary blood flow expressed as maximum standardized uptake values in the injury group $(n=5)$ and control group $(n=6)$ for each ROI. Values represent the median [interquartile range]. $P$ values were calculated to compare each ROI of the left and right lung within the control group and between the injured and contralateral lungs within the acid aspiration group.

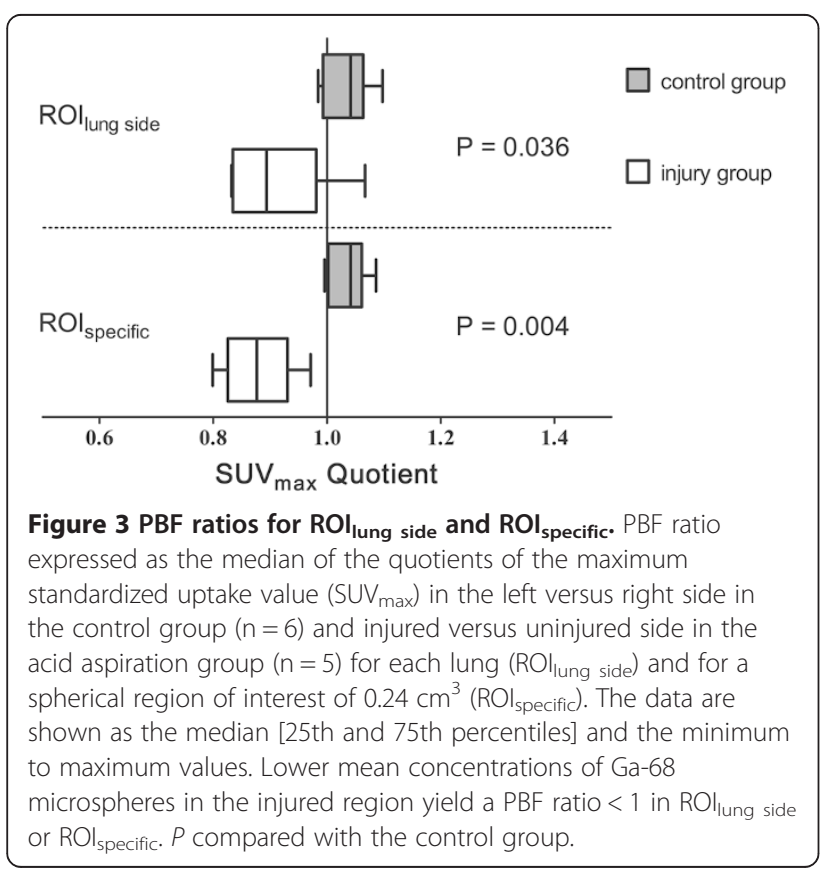

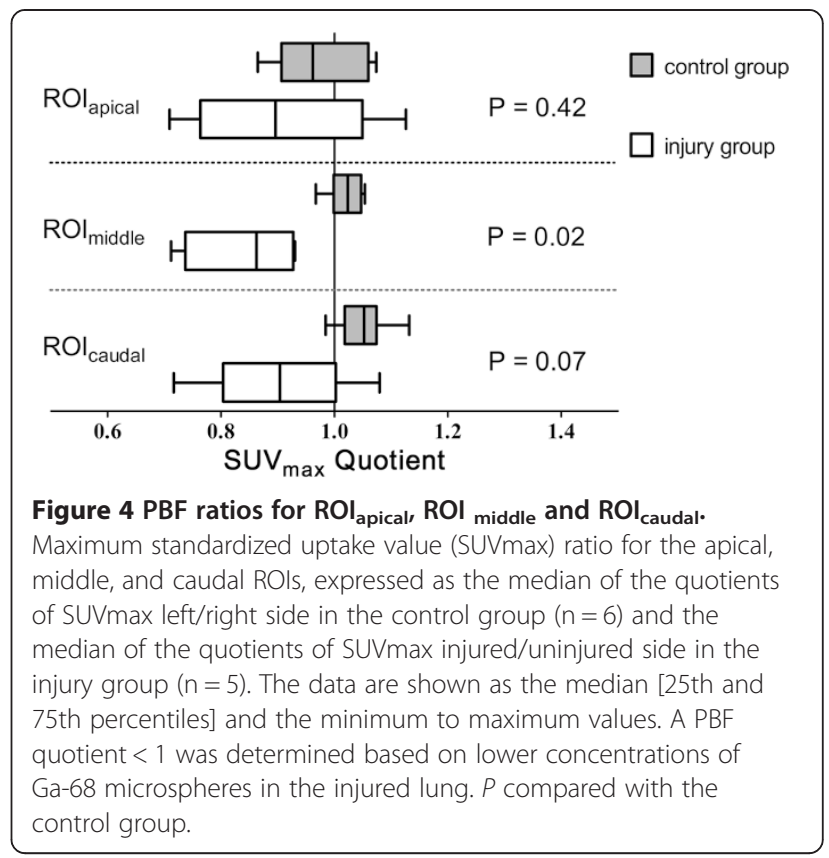

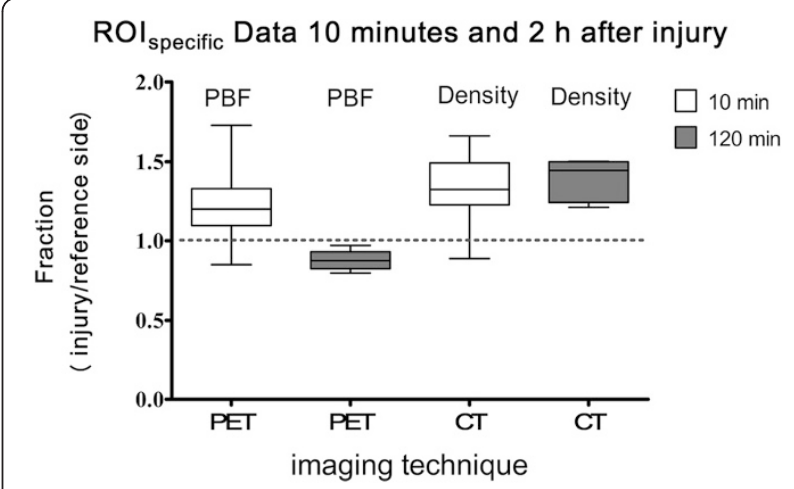

Figure $5 \mathrm{PBF}$ and density ratios for $\mathrm{ROI}_{\text {specific }}$. Data from PET and $\mathrm{CT}$ images representing the PBF and density fraction of injured to uninjured lung for a region of interest $\left(\mathrm{ROI}_{\text {specific }}\right)$ in all of the injured animals in the present study ( 2 hours after injury; $n=5$ ) and a previous study $(n=12)[11]$ in which the spatial PBF distribution was measured 10 minutes after acid aspiration injury. The data are shown as the median [25th and 75th percentiles] and the minimum to maximum values. The $\mathrm{ROI}_{\text {specific }}$ was spherical and of identical size $\left(0.24 \mathrm{~cm}^{3}\right)$ for all animals and for the PET and CT images of each animal. For the mean density values (CT images) and for the mean radioactivity concentration values (PET images), the presented fractions were calculated using the quotient of the $\mathrm{ROI}_{\text {specific }}$ values for the injured side versus that of the non-injured side of each animal. A greater mean density on the injured side resulted in a $\mathrm{CT}$ quotient $>1$ in the $\mathrm{ROI}_{\text {specific. }}$ Lower mean concentrations of Ga-68 microspheres on the injured side 2 hours after injury yielded a PET

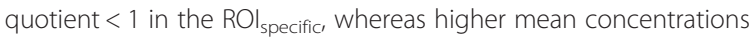
of Ga-68 microspheres on the injured side 10 minutes after injury yielded a PET quotient $>1$ in the ROI specific. $_{\text {. }}$ 


\section{Acid aspiration}

Acute lung injury was induced with a small amount of $\mathrm{HCl}$, which is associated with a low mortality rate [17]. While the physiological parameters remained stable in the control animals, acid-induced lung injury caused deterioration of $\mathrm{PaO}_{2}$ and $\mathrm{SpaO}_{2}$ values shortly after the administration of $\mathrm{HCl}$. These parameters recovered to values that were not significantly different from those of the control group at 110 minutes after injury; however, $\mathrm{PaO}_{2}$ did not reach baseline levels. In line with previous observations [11], acid injury induced hypotension. Although hypoxemia improved over time, the arterial blood pressure did not. The heart rate remained unchanged during the observation period, which was consistent with findings reported in the literature $[9,11]$.

Contact with concentrated acid forms membrane pores in the alveolar epithelium and initiates a sequence of events that results in the recruitment of leukocytes and the formation of edema [18]. Within 4 hours after administration, $\mathrm{HCl}$ caused cellular injury, which has been characterized by an increased number of intra-alveolar erythrocytes, the recruitment of neutrophils, and fibrin formation $[13,19]$. The impact of acid on lung tissue is represented by an increase in tissue density in the injured areas [20,9]. Using CT scans to detect lung density changes [21], we found that almost all of the animals were injured in the middle and caudal areas, which was anticipated based on bronchial anatomy. The density ratio (injury/reference side of $\mathrm{ROI}_{\text {specific }}$ ) obtained 2.5 hours after injury in this study (1.4 [1.2-1.5]) was comparable to the density ratio observed within 1 hour after acid administration in rats (1.3 [1.2;1.5]) in a previous study [11] (Figure 5).

\section{PBF}

Data regarding the effects of acid aspiration on regional PBF are limited [11,22]. We observed different PBF ratios between the injury and control groups that demonstrated decreased PBF on the injured side compared with the noninjured side in the aspiration group. This difference was the result of changes on either side, namely, a non-significant increase in PBF on the non-injured side and a nonsignificant decrease in PBF on the injured side (Table 2). Additionally, these findings indicate a redistribution of lung perfusion away from injured regions over time. The location of this redistribution differs from that observed within the first hour after aspiration [11,12]. In fact, contrary to the results of the present study, the very early phase (10 minutes after acid aspiration injury in rats) was characterized by a redistribution of perfusion toward regions of increased density, with higher PBF ratios between the injured and the uninjured lung sides in $\mathrm{ROI}_{\text {specific }}$ in the aspiration group compared with the control group (Figure 5) [11].

When comparing 3 transverse ROIs, the PBF was different in areas where the $\mathrm{HCl}$ produced the most abundant density changes, namely, the middle region. Again, the uninjured side showed a non-significantly higher PBF than the injured side, which resulted in a significantly different $\mathrm{PBF}$ ratio in the $\mathrm{ROI}_{\text {middle }}$ in the injured animals compared with the control group (Figure 4). This finding is contrary to observations from the early stage of acid-induced injury, where the PBF ratio was different for the apical, middle, and caudal ROIs because of increased PBF on the injured side [11].

Multiple factors may be responsible for the reduction of $\mathrm{PBF}$ in injured regions during the later stages of aspiration-induced ALI. The spatial distribution of PBF is dependent on oxygen tension [23], and experimental hypoxia can cause changes in flow distribution [24-26]. The hypoxic pulmonary vasoconstriction may have been blunted or blocked by the acid injury [27] and may have been further limited by relaxing factors that were released from the endothelium in the pulmonary vessels during hypoxia [28] in the early stages of the inflammatory response (10 minutes after acid aspiration). Those factors may have been compensated for over time, resulting in hypoxic pulmonary vasoconstriction 2 hours after injury. Otherwise, the initial hyperperfusion in injured regions may have led to additional alveolar damage [29]. Therefore, it can be speculated that the increased PBF in aspiration areas could promote a loss of fluid through the damaged endothelium with subsequent regional edema. Additionally, the stepwise development of alveolar hemorrhage, intra-alveolar edema, and interstitial edema [30,31] after acid administration in rats is an inflammatory response that depends on circulating mediators $[13,32,33]$. The edema formation, with resulting extravascular pressure in the lung parenchyma, may reduce the spatial PBF in damaged areas over time.

Pulmonary perfusion distribution has been examined in different models of acute lung injury and has been reported to change based on position [34-37] or interventions in the ventilator setting $[38,37,39]$. To study the changes in PBF in the aspiration model used in this study, intra-individual comparisons of PBF are possible.

Beginning from the first minute after acid aspiration in spontaneously breathing rats, damaged regions are preferentially perfused within the first hour after injury $[11,12]$. The fact that the PBF decreases as the inflammatory process continues shows that regional hyperperfusion in regions of acid induced lung damage is limited over time. Therefore, interventions such as targeted drug delivery do not preferentially reach areas of lung injury after 2 hours in this model of acute lung injury. However, it remains unclear whether the PBF distribution pattern in this model is relevant to other types of lung injury or to other species.

Lung injury such as acid aspiration-induced injury, blunt trauma/lung contusion and bacterial inflammation, which can occur in isolated parts of the lung, may show 
completely different perfusion distribution in a certain time frame, depending on the degree and onset of increased intravascular permeability, interstitial edema, neutrophilic alveolitis [19] and the therapeutic interventions such as ventilation. Therefore, the PBF distribution pattern requires further investigation in different animal models and over time.

\section{Limitations of the study}

In this model of anesthetized, spontaneously breathing rats, we considered the effects of acid alone, in the absence of particulate matter, which is commonly a component of aspirated fluid in humans. In humans, aspiration frequently affects the lower right pulmonary region; however, the fact that the left side was most affected in this study may represent aspiration events that can occur with a spatial alignment different from the supine or right position. This study focused on the PBF distribution between the first and second peaks of the inflammatory response in rats [13]. Potentially underlying mechanisms for the PBF pattern, such as endothelial damage, cardiac output, edema formation, platelet aggregation, and changes in regional ventilation, were not considered.

The number of animals used in the current study when assessing differences in the $\mathrm{SUV}_{\max }$ ratios was small but with an adequate power for the statements made (especially for $\mathrm{ROI}_{\text {specific }}$ and $\left.\mathrm{ROI}_{\text {middle }}\right)$. To better reflect clinical experience, further studies are needed to obtain information about the PBF distribution pattern in cases in which greater aspiration involves a larger volume of injured lung. Additionally, the possible need for ventilatory support in cases of massive aspiration necessitates studies examining the influence of positive pressure ventilation (in different settings) on PBF. Interestingly, a higher proportion of spontaneous breathing reduced lung injury as compared with protective mechanical ventilation in a surfactant depletion model [40] or after acid aspiration [41]. In the current study, the PET images represent the PBF during the entire breathing cycle in spontaneously breathing animals. The breathing pattern was continuously monitored based on the respiratory rate only, because depression of ventilation in spontaneously breathing rats was recognized to be due to a depression in the respiratory rate, as discussed previously [15]. Therefore, the absence of changes in the respiratory rate suggests stable respiration during the experiment.

The standardized acid aspiration model and the PET technique used in the present study have been previously discussed [11,15]. We used a 30-minute data acquisition time for the emission scan to optimize the activity count rate; the influence of potential density changes during the PET scan on PBF, obtained from volume data, has been shown to not significantly influence the results [11].

\section{Conclusions}

In this experimental rat model of acid aspirationinduced lung injury, we observed a decrease in PBF in injured lung regions compared with uninjured regions 2 hours after the aspiration event. This redistribution of pulmonary perfusion away from injured regions likely accounted for the partial recovery from hypoxemia that was observed at 110 minutes compared with 20 minutes after injury. Taken together, the results of the present and previous studies [11,12], which covered the time window of 2 hours post aspiration, suggest that an initial shift of perfusion toward injured regions after aspiration is followed by redistribution away from them. The potential clinical implication of these experimental findings is that intravenous treatments may not preferentially reach injured lung regions unless they are administered within a specific time during the first hour of the aspiration event.

\section{Abbreviations}

ALI: Acute lung injury; $\mathrm{CT}$ : Computed tomography; $\mathrm{HCl}$ : Hydrochloric acid; $\mathrm{PaO}_{2}$ : Arterial oxygen tension; PBF: Pulmonary blood flow; PET: Positron emission tomography; $\mathrm{ROI}$ : Region of interest; $\mathrm{SpaO}_{2}$ : Arterial oxygen saturation; SUV: Standardized uptake value.

\section{Competing interests}

The authors declare that they have no competing interests.

\section{Authors' contributions}

TR and RB conceived of the study and performed the experiments. TR, RB, GM and JP participated in the image processing. TR, GM and RB participated in the data analysis and interpretation. TR, TK, JP and GM participated in the study design and coordination and helped to draft the manuscript. All authors read and approved the final manuscript.

\section{Authors' information}

TR: Anesthetist, Senior Physician.

RB: Senior Scientist.

GM: Associate Professor of Anesthesia, Department of Anesthesia, Critical

Care and Pain Medicine.

JP: Professor, Head of the Department of Radiopharmaceutical and Chemical Biology.

TK: Professor and Chair of the Department of Anesthesia and Intensive Care.

\section{Acknowledgments}

We would like to thank Regina Herrlich, Natalie Thieme, and Andrea Suhr, who are staff technologists in the Department of Radiopharmaceutical and Chemical Biology, Institute of Radiopharmaceutical Cancer Research, Helmholtz-Zentrum Dresden-Rossendorf, Dresden, Germany, Uta Schwanebeck, staff statistician, Coordination Centre for Clinical Trials and Maximilian Ragaller, M.D., Professor and Head of the Intensive Care Unit, Department of Anesthesia and Intensive Care, Carl Gustav Carus University Hospital at the Technische Universität Dresden, Dresden, Germany.

Funding support was provided by institutional and departmental sources. Guido Musch was supported by grant R01HL094639 from the National Institutes of Health. The study was conducted at Helmholtz-Zentrum Dresden-Rossendorf, Dresden, Germany.

\section{Author details}

${ }^{1}$ Department of Anesthesia and Intensive Care, Carl Gustav Carus University Hospital, Technische Universität Dresden, Dresden, Germany. ${ }^{2}$ Department of Radiopharmaceutical and Chemical Biology, Institute of Radiopharmaceutical Cancer Research, Helmholtz-Zentrum Dresden- Rossendorf, Dresden, Germany. ${ }^{3}$ Department of Anesthesia, Critical Care and Pain Medicine, Massachusetts General Hospital and Harvard Medical School, Boston, Massachusetts, USA. ${ }^{4}$ Department of Chemistry and Food Chemistry, Technische Universität Dresden, Dresden, Germany. 
Received: 16 November 2014 Accepted: 24 February 2015 Published online: 18 March 2015

\section{References}

1. Knight PR, Rutter T, Tait AR, Coleman E, Johnson K. Pathogenesis of gastric particulate lung injury: a comparison and interaction with acidic pneumonitis. Anesth Analg. 1993;77(4):754-60.

2. Raghavendran K, Nemzek J, Napolitano LM, Knight PR. Aspiration-induced lung injury. Crit Care Med. 2011;39(4):818-26.

3. Marik PE. Aspiration pneumonitis and aspiration pneumonia. N Engl J Med. 2001;344(9):665-71.

4. Marrie TJ, Carriere KC, Jin Y, Johnson DH. Factors associated with death among adults $<55$ years of age hospitalized for community-acquired pneumonia. Clin Infect Dis. 2003;36(4):413-21.

5. Rubenfeld GD, Caldwell E, Peabody E, Weaver J, Martin DP, Neff M, et al. Incidence and outcomes of acute lung injury. N Engl J Med. 2005;353 (16):1685-93.

6. Dudley WR, Marshall BE. Steroid treatment for acid-aspiration pneumonitis. Anesthesiology. 1974;40(2):136-41.

7. Safdar Z, Yiming M, Grunig G, Bhattacharya J. Inhibition of acid-induced lung injury by hyperosmolar sucrose in rats. Am J Respir Crit Care Med. 2005;172(8):1002-7.

8. Rabinovici R, Vernick J, Hillegas L, Neville LF. Hypertonic saline treatment of acid aspiration-induced lung injury. J Surg Res. 1996;60(1):176-80.

9. Pawlik MT, Schreyer AG, Ittner KP, Selig C, Gruber M, Feuerbach S, et al. Early treatment with pentoxifylline reduces lung injury induced by acid aspiration in rats. Chest. 2005;127(2):613-21.

10. Grimbert FA, Parker JC, Taylor AE. Increased pulmonary vascular permeability following acid aspiration. J Appl Physiol. 1981;51(2):335-45.

11. Richter T, Bergmann R, Knels L, Hofheinz F, Kasper M, Deile M, et al. Pulmonary blood flow increases in damaged regions directly after acid aspiration in rats. Anesthesiology. 2013;119(4):890-900.

12. Richter T, Bergmann R, Pietzsch J, Mueller MP, Koch T. Effects of pulmonary acid aspiration on the regional pulmonary blood flow within the first hour after injury: an observational study in rats. Clin Hemorheol Microcirc. 2014. doi:10.3233/CH-141867.

13. Kennedy TP, Johnson KJ, Kunkel RG, Ward PA, Knight PR, Finch JS. Acute acid aspiration lung injury in the rat: biphasic pathogenesis. Anesth Analg. 1989;69(1):87-92.

14. Nanjo S, Bhattacharya J, Staub NC. Concentrated albumin does not affect lung edema formation after acid instillation in the dog. Am Rev Respir Dis. 1983;128(5):884-9.

15. Richter T, Bergmann R, Pietzsch J, Kozle I, Hofheinz F, Schiller E, et al. Effects of posture on regional pulmonary blood flow in rats as measured by PET. J Appl Physiol. 2010;108(2):422-9.

16. Vieira SR, Puybasset L, Richecoeur J, Lu Q, Cluzel P, Gusman PB, et al. A lung computed tomographic assessment of positive end-expiratory pressure-induced lung overdistension. Am J Respir Crit Care Med. 1998;158(5 Pt 1):1571-7.

17. James CF, Modell JH, Gibbs CP, Kuck EJ, Ruiz BC. Pulmonary aspiration-effects of volume and $\mathrm{pH}$ in the rat. Anesth Analg. 1984;63 (7):665-8.

18. Westphalen K, Monma E, Islam MN, Bhattacharya J. Acid contact in the rodent pulmonary alveolus causes proinflammatory signaling by membrane pore formation. Am J Physiol Lung Cell Mol Physiol. 2012;303(2):107-16.

19. Matute-Bello G, Frevert CW, Martin TR. Animal models of acute lung injury. Am J Physiol Lung Cell Mol Physiol. 2008;295(3):379-99.

20. Lim YS, Chung MH, Park SH, Kim HY, Choi BG, Lim HW, et al. Acute and repeated inhalation lung injury by 3-methoxybutyl chloroformate in rats: CT-pathologic correlation. Eur J Radiol. 2007;62(2):227-34.

21. Routly JJ, Puybasset L, Nieszkowska A, Lu Q. Acute respiratory distress syndrome: Lessons from computed tomography of the whole lung. Crit Care Med. 2003;31 (4):S285-95.

22. Carlile PV, Hagan SF, Gray BA. Perfusion distribution and lung thermal volume in canine hydrochloric acid aspiration. J Appl Physiol. 1988;65 (2):750-9.

23. Melsom MN, Flatebo T, Nicolaysen G. Hypoxia and hyperoxia both transiently affect distribution of pulmonary perfusion but not ventilation in awake sheep. Acta Physiol Scand. 1999;166(2):151-8.

24. Hlastala MP, Lamm WJ, Karp A, Polissar NL, Starr IR, Glenny RW. Spatial distribution of hypoxic pulmonary vasoconstriction in the supine pig. J Appl Physiol. 2004;96(5):1589-99.
25. Lamm WJ, Starr IR, Neradilek B, Polissar NL, Glenny RW, Hlastala MP. Hypoxic pulmonary vasoconstriction is heterogeneously distributed in the prone dog. Respir Physiol Neurobiol. 2004;144(2-3):281-94.

26. Starr IR, Lamm WJ, Neradilek B, Polissar N, Glenny RW, Hlastala MP. Regional hypoxic pulmonary vasoconstriction in prone pigs. J Appl Physiol. 2005;99 (1):363-70.

27. Schuster DP, Anderson C, Kozlowski J, Lange N. Regional pulmonary perfusion in patients with acute pulmonary edema. J Nucl Med. 2002;43 (7):863-70.

28. Brashers VL, Peach MJ, Rose Jr CE. Augmentation of hypoxic pulmonary vasoconstriction in the isolated perfused rat lung by in vitro antagonists of endothelium-dependent relaxation. J Clin Invest. 1988;82(5):1495-502.

29. Kozian A, Schilling T, Freden F, Maripuu E, Rocken C, Strang C, et al. Onelung ventilation induces hyperperfusion and alveolar damage in the ventilated lung: an experimental study. Br J Anaesth. 2008;100(4):549-59.

30. McAuley DF, Frank JA, Fang XH, Matthay MA. Clinically relevant concentrations of beta(2)-adrenergic agonists stimulate maximal cyclic adenosine monophosphate-dependent airspace fluid clearance and decrease pulmonary edema in experimental acid-induced lung injury. Crit Care Med. 2004;32(7):1470-6.

31. Zarbock A, Allegretti M, Ley K. Therapeutic inhibition of CXCR2 by Reparixin attenuates acute lung injury in mice. Brit J Pharmacol. 2008;155(3):357-64.

32. Folkesson HG, Matthay MA, Hebert CA, Broaddus VC. Acid aspirationinduced lung injury in rabbits is mediated by interleukin-8-dependent mechanisms. J Clin Invest. 1995;96(1):107-16.

33. Knight PR, Druskovich G, Tait A, Johnson K. The role of neutrophils, oxidants and proteases in pulmonary acid injury. Anesthesiology. 1992;77(4):772-8.

34. Wiener CM, Kirk W, Albert RK. Prone position reverses gravitational distribution of perfusion in dog lungs with oleic acid-induced injury. J Appl Physiol. 1990;68(4):1386-92.

35. Lamm WJ, Graham MM, Albert RK. Mechanism by which the prone position improves oxygenation in acute lung injury. Am J Respir Crit Care Med. 1994;150(1):184-93.

36. Richter T, Bellani G, Scott Harris R, Vidal Melo MF, Winkler T, Venegas JG, et al. Effect of prone position on regional shunt, aeration, and perfusion in experimental acute lung injury. Am J Respir Crit Care Med. 2005;172(4):480-7.

37. Richard JC, Bregeon F, Costes N, Bars DL, Tourvieille C, Lavenne F, et al. Effects of prone position and positive end-expiratory pressure on lung perfusion and ventilation. Crit Care Med. 2008;36(8):2373-80.

38. Musch G, Harris RS, Vidal Melo MF, O'Neill KR, Layfield JD, Winkler T, et al. Mechanism by which a sustained inflation can worsen oxygenation in acute lung injury. Anesthesiology. 2004;100(2):323-30.

39. Guldner A, Kiss T, Bluth T, Uhlig C, Braune A, Carvalho N, et al. Effects of Ultraprotective Ventilation, Extracorporeal Carbon Dioxide Removal, and Spontaneous Breathing on Lung Morphofunction and Inflammation in Experimental Severe Acute Respiratory Distress Syndrome. Anesthesiology. 2014. doi:10.1097/ALN.0000000000000504.

40. Carvalho NC, Guldner A, Beda A, Rentzsch I, Uhlig C, Dittrich S, et al. Higher levels of spontaneous breathing reduce lung injury in experimental moderate acute respiratory distress syndrome. Crit Care Med. 2014;42 (11):702-15.

41. Xia J, Zhang H, Sun B, Yang R, He H, Zhan Q. Spontaneous breathing with biphasic positive airway pressure attenuates lung injury in hydrochloric acid-induced acute respiratory distress syndrome. Anesthesiology. 2014;120 (6):1441-9. 